\title{
Ionization Mechanism of the Ambient Pressure Pyroelectric Ion Source (APPIS) and Its Applications to Chemical Nerve Agent Detection
}

\author{
Evan L. Neidholdt and J. L. Beauchamp \\ Noyes Laboratory of Chemical Physics and the Beckman Institute, California Institute of Technology, \\ Pasadena, California, USA
}

We present studies of the ionization mechanism operative in the ambient pressure pyroelectric ionization source (APPIS), along with applications that include detection of simulants for chemical nerve agents. It is found that ionization by APPIS occurs in the gas-phase. As the crystal is thermally cycled over a narrow temperature range, electrical discharges near the surface of the crystal produce energetic species which, through reactions with atmospheric molecules, result in reactant ions such as protonated water clusters or clusters of hydroxide and water. Reactant ions can be observed directly in the mass spectrometer. These go on to react with trace neutrals via proton transfer reactions to produce the ions observed in mass spectra, which are usually singly protonated or deprotonated species. Further implicating gas-phase ionization, observed product distributions are highly dependent on the composition of ambient gases, especially the concentration of water vapor and oxygen surrounding the source. For example, basic species such as triethylamine are observed as singly protonated cations at a water partial pressure of 10 torr. At a water pressure of 4 torr, reactive oxygen species are formed and lead to observation of protonated amine oxides. The ability of the APPIS source to detect basic molecules with high proton affinities makes it highly suited for the detection of chemical nerve agents. We demonstrate this application using simulants corresponding to VX and GA (Tabun). With the present source configuration pyridine is detected readily at a concentration of $4 \mathrm{ppm}$, indicating ultimate sensitivity in the high $\mathrm{ppb}$ range. (J Am Soc Mass Spectrom 2009, 20, 2093-2099) (c) 2009 American Society for Mass Spectrometry

$\mathrm{P}$ reviously [1] we presented the design and implementation of a novel ambient pressure pyroelectric ion source (APPIS) for mass spectrometry. The source comprises a thermally cycled pyroelectric crystal (lithium niobate or lithium tantalate) coupled with a neutral vapor source in front of the atmospheric pressure inlet of an ion trap mass spectrometer. Ions are produced at or near the crystal surface during periods of thermal cycling, and positive and negative ions are alternately produced upon heating and cooling, respectively, at the $-\mathrm{z}$ face. Fragment ions are typically not observed in the mass spectrum, revealing that APPIS ionization is a "soft" ionization method. Acidic and basic species are typically observed as the corresponding deprotonated and protonated molecular ions, respectively.

Electrical discharges are observed at the faces of the crystal during thermal cycling, and we suggested that they may contribute to the detected ion signal. Ion sources using some form of electrical discharge are numerous and well-known, including various forms of

Address reprint requests to Dr. J. L. Beauchamp, Noyes Laboratory of Chemical Physics and the Beckman Institute, California Institute of Technology, Mail Code 127-72, Pasadena, CA 91125, USA. E-mail: jlbchamp@ caltech.edu glow discharge ionization [2-4] and corona discharge ionization [5]. Dielectric barrier discharge ionization [6] creates an AC discharge between a needle and a thin dielectric layer, under which lies an electrode that completes the discharge circuit, and ions can be desorbed from this dielectric layer and sampled by mass spectrometry. Since discharges may play an important part in APPIS ionization, we present here an investigation into the nature of the observed discharges and their relationship to ion production. A mechanism of ionization involving a form of atmospheric pressure chemical ionization is proposed, whereby molecules that are detected as protonated species have undergone reaction with reactant ions present in humid air of the form ${ }^{+} \mathrm{H}\left(\mathrm{H}_{2} \mathrm{O}\right)_{n}$ or ${ }^{-} \mathrm{OH}\left(\mathrm{H}_{2} \mathrm{O}\right)_{n}$. Previous work by others on pyroelectric materials has revealed the production of ions and electrons from pyroelectric crystals [7-15] as well as field ionization of deuterium gas to form $\mathrm{D}^{+}$, which is used in pyroelectric fusion experiments [16, 17]. None of these studies involve ionization at ambient pressure as the chief mode of ionization.

In addition to studies of the ionization mechanism, we investigate the utility of the source for the detection of simulants for chemical nerve agents. It was seen previously that volatile organic molecules with basic 
functional groups were detected as protonated species using APPIS mass spectrometry [1]. Numerous chemical nerve agents contain basic functional groups and are likely well suited to detection by APPIS mass spectrometry. Previous investigations that have reported the detection of chemical nerve agents by ion mobility and mass spectrometry [18-20] use compounds similar in reactivity to the nerve agents, termed simulants. Diethyl phosphoramidate (DEPA) simulates Tabun (GA), and 2-(butylamino)ethanethiol simulates VX. We demonstrate the detection of these nerve agent simulants using APPIS and ion trap mass spectrometry. Lastly, a sensitivity study was performed using pyridine, and it was found that pyridine is readily detectable down to concentrations of 4 parts per million, suggesting ultimate sensitivity of the current source implementation in the high part per billion range.

\section{Experimental}

Figure 1a shows a schematic of APPIS, which is installed in front of the atmospheric pressure inlet of an ion trap mass spectrometer (LCQ Deca, Thermo Scientific, San Jose, CA, USA). For the experiments described here, a $5 \times 5 \times 5 \mathrm{~mm}$ pyroelectric $\mathrm{LiTaO}_{3}$ crystal (Elan Ltd., St. Petersburg, Russia) is mounted with the $-z$ face exposed and a resistance heater attached to the $+\mathrm{z}$ face. The heater comprises a $62 \Omega, 0.5 \mathrm{~W}$ resistor epoxied to the crystal with Arctic Alumina thermal adhesive (Arctic Silver Inc., Visalia, CA, USA). Temperature is measured with a copper constantan thermocouple, interfaced with LabVIEW (National Instruments, Austin, TX, USA) by a National Instruments CompactDAQ thermocouple module. The proposed ionization mechanism, discussed in further detail below, is depicted in Figure 1b, and involves electrical discharges at the crystal face. To monitor electrical activity on the crystal face, the source was additionally fitted with an inductive pickup. The pickup comprises a stainless steel ring, to which an electrical connection was made, suspended inside the vapor containment shroud by support wires, $4 \mathrm{~mm}$ from and centered with the -z crystal face. The pickup is electrically insulated from the shroud and connected through a current measuring device to ground. Either a Keithley 485 autoranging picoammeter having a measured time constant of $\tau=2 \mathrm{~ms}$ or an operational amplifier having a gain of $10^{7} \mathrm{~V} / \mathrm{A}$ and measured time constant of $\tau=100 \mu \mathrm{S}$ was used for current measurement. The picoammeter afforded better sensitivity to lower current levels, at the cost of an increased circuit response time. The operational amplifier, although less sensitive, allowed for better resolution of temporal features in the measured induction current. Induction current is the flow of charge induced on the pickup by the charged crystal face, detected as a current in the external circuit. The sign of the current measured is the same as the charge developed on the crystal face when current is defined as the flow of positive charge. The voltage outputs of the picoammeter and op-amp were pyroelectric crystal
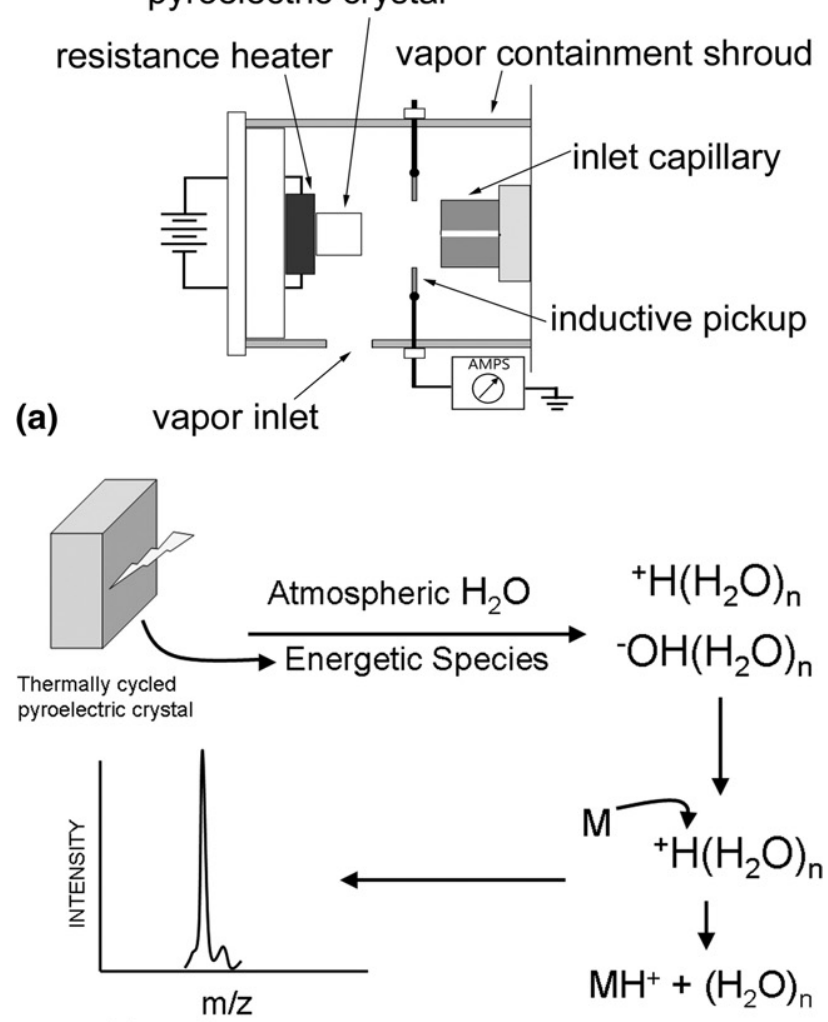

(b) Mass Spectrum

Proton Xfer Rxns

(c)

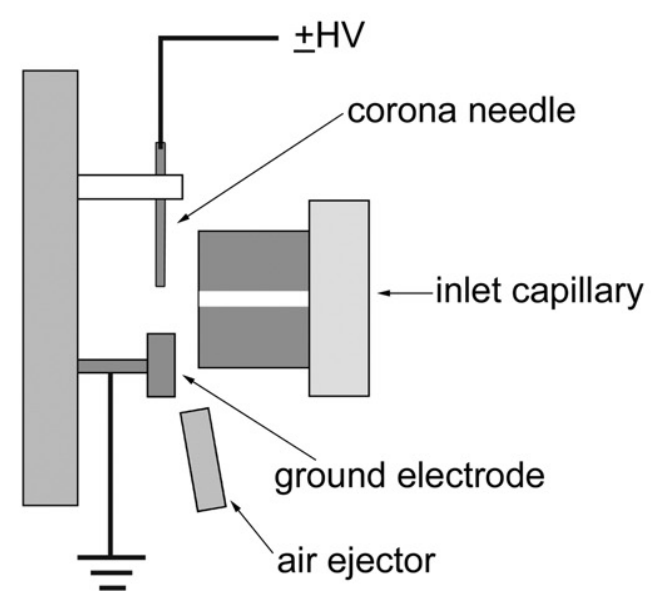

Figure 1. (a) Schematic of APPIS source (to scale). Ions are produced at the $-\mathrm{z}$ face of a pyroelectric crystal and travel through the vapor containment shroud to the atmospheric pressure inlet capillary of the mass spectrometer. The crystal is heated by a resistor cemented to the $+\mathrm{z}$ face. An inductive pickup is placed so that induction current (that is, the flow of charge induced on the electrode because of the temporal variation in charge on the crystal face) can be monitored. (b) Proposed ionization mechanism for APPIS. Electrical discharge on the crystal surface leads to the production of energetic species, which combine and react with atmospheric water to create reagent ions that are commonly protonated water clusters or clusters of hydroxide anion and water. These reactant ions take part in proton transfer reactions with basic and acidic species, leading to ionization of the analytes and observation of them in the mass spectrometer. (c) Schematic of the corona discharge source. High voltage is applied to the needle and the corona discharge occurs between the needle and the ground electrode. Sample is introduced via the pneumatic air ejector. 
monitored and recorded using LabVIEW with an NI9205 CompactDAQ input module (National Instruments, Austin, TX, USA).

Figure $1 \mathrm{c}$ is a schematic of an atmospheric pressure chemical ionization (APCI) source, which was constructed to compare ionization by this method to APPIS ionization. The APCI source comprised a direct current (DC) corona discharge in air placed in close proximity to the atmospheric pressure inlet of a ThermoFinnigan LCQ Deca ion trap mass spectrometer. An inlet capillary extension was added to the instrument in this case, so that the corona could be easily placed within $5 \mathrm{~mm}$ of the ion sampling inlet. A tungsten inert gas welding electrode, ground to a point, was used with a gap distance of 1-2 $\mathrm{mm}$ between the electrode point and ground. A starting potential of between 1500-3000 V was applied to the needle, which was connected to the high voltage power supply through a $10 \mathrm{M} \Omega, 1 \mathrm{~W}$ current limiting resistor. Samples for APCI analysis were introduced into the corona using a fabricated pneumatic air ejector. To operate the ejector, compressed air expands from a small orifice, which causes a reduced pressure region near the sample inlet, causing sample to be mixed with air. The air/sample mixture is exhausted into the corona, and the ions produced are sampled through the atmospheric inlet capillary of the mass spectrometer.

Samples for determination of detection limits were prepared by on-line dilution using a model 1010 gas diluter (Custom Sensor Solutions, Oro Valley, AZ, USA). Samples of pyridine were prepared by injecting 10-25 $\mu \mathrm{L}$ liquid pyridine into a $40 \mathrm{~L}$ capacity Tedlar sample bag (Zefon International, Ocala, FL, USA), which was then filled with $40 \mathrm{~L}$ of dry nitrogen. Two hundred $\mu \mathrm{L}$ water was additionally injected into the bag to set a water partial pressure of $\sim 6$ torr $(800 \mathrm{~Pa})$, emulating atmospheric conditions. The sample bag was then connected to the sample input of the gas diluter, whose output was then connected to the gas inlet port of the APPIS source. Dilutions were performed with a diluent bag also containing nitrogen and water. The gas diluter has useable dilution settings from $2 \%$ to $100 \%$, meaning that available concentration ranged from $2 \%$ to $100 \%$ of the prepared concentration.

Triethylamine and pyridine were from Fisher Scientific, Waltham, MA, USA. Hexafluorobenzene and 1,1,1,3,3,3-hexafluoroisopropanol were from Fluka (sold by Sigma-Aldrich, St. Louis, MO, USA); 2-(butylamino)ethanethiol, diethylphosphoramidate, and benzoic acid were from Sigma-Aldrich. All chemicals were used as received, without further purification.

\section{Results and Discussion}

Ions are observed in coincidence with electrical activity as detected by the inductive pickup. Figure 2a exhibits the temporal variation of induction current and total ion current as detected by the mass spectrometer during an experiment where the crystal is being heated, pro-
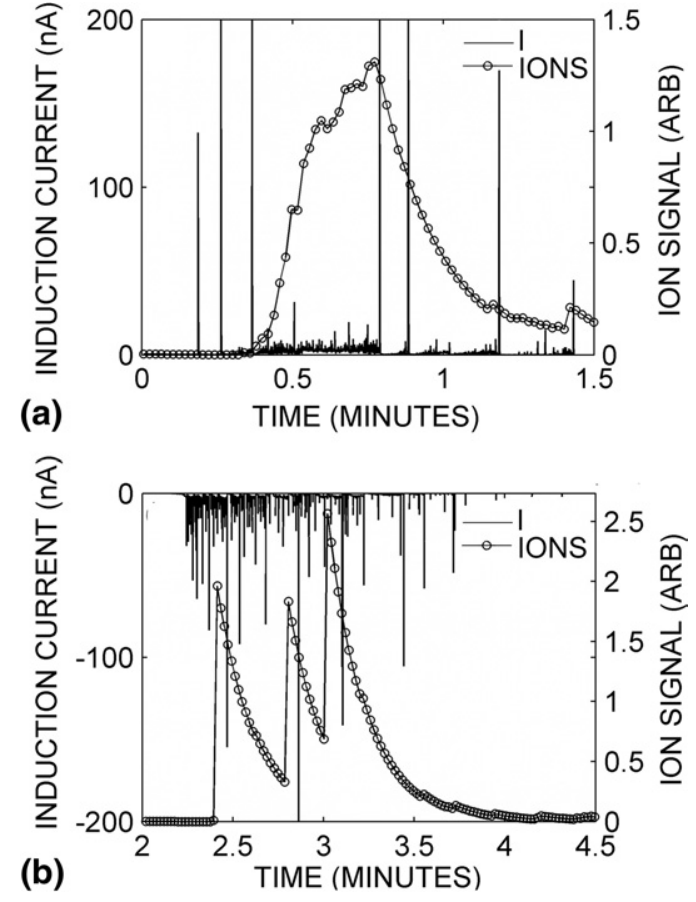

Figure 2. (a) Temporal variation of ion signal and induction current for positive ion formation from triethylamine. The crystal was heated at a rate of $\sim 0.5 \mathrm{~K} \mathrm{~s}^{-1}$, from $t=0$ to $t=1.5 \mathrm{~min}$. The frequency of spark discharges is low relative to the lower intensity dark discharging, and most ions are produced during periods of dark discharging. (b) Temporal variation of ion signal and induction current for negative ions from hexafluoroisopropanol. The crystal was first heated to a temperature of $\sim 80^{\circ} \mathrm{C}$, and then allowed to cool at a rate of $0.5 \mathrm{~K} \mathrm{~s}^{-1}$. As further discussed in this text, the frequency of sparking is high compared with that observed when the crystal is heated. Ion production occurs during the periods of electrical activity.

ducing cations from triethylamine in air. In the present implementation, ion production appears to be strongly correlated with the more frequent, lower current discharges. Figure $2 b$ illustrates the variation with time of induction current and total ion current for an experiment, where anions from 1,1,1,3,3,3-hexafluoroisopropanol are detected. The crystal was first heated to a temperature near $80^{\circ} \mathrm{C}$ (region not shown, from $t=0$ to $\mathrm{t}=2 \mathrm{~min})$ and allowed to cool $(\mathrm{t}=2$ to $\mathrm{t}=6 \mathrm{~min}$, shown). In the anionic experiments, there are many more of the higher current discharges occurring during the time of ion production. This is likely due to the presence of electrons at the crystal surface, a consequence of the pyroelectric effect [14]. These electrons "prime" the air with excess charge so that dielectric breakdown occurs more easily than in the case of a positively charged crystal. The discharges in each case correspond to a buildup of charge up to the point of dielectric breakdown, followed by a rapid flow of this charge to a region nearer to ground potential, either the opposite crystal face, a part of the metal mount near the crystal, or another region on the crystal face. Electrical discharges at the crystal face leading to ionization of analyte suggest a gas-phase ionization mechanism. An 
APCI source was constructed to compare the results of ionization by stand-alone APCI with those of APPIS.

The APCI mass spectra of test compounds were essentially identical to the APPIS mass spectra of the same compounds when acquired under similar environmental conditions. Differences observed in the APCI and APPIS mass spectra can be accounted for by considering differences in gas composition associated with each experiment. APCI experiments were performed by injecting the sample into the corona using compressed air, which contains very little water vapor because it is dried during production so as not to condense water vapor during the compression process. Any pneumatically assisted APCI experiments using our corona discharge source will take place in relatively dry air. Owing to the ambient pressure characteristics and projected future use of the source, APPIS experiments are typically performed by analyzing the sample vapor in an environment of ordinary laboratory air, which is on average between $40 \%$ and $60 \%$ relative humidity, as determined by dew point measurements. This corresponds to a partial pressure of water in air of about 10 torr $\left(1.3 \times 10^{3} \mathrm{~Pa}\right)$. Under the typical conditions for each experiment (dry air for APCI, humid air for APPIS), the mass spectra for triethylamine show distinctly different product yields.

APPIS mass spectra of triethylamine on typical day $\left(\mathrm{p}\left(\mathrm{H}_{2} \mathrm{O}\right)=10\right.$ torr, $\left.1.3 \times 10^{3} \mathrm{~Pa}\right)$ yield the protonated molecular ion as the base peak in the spectrum and a small percentage of what we assign as the protonated n-oxide of triethylamine. Figure $3 a$ shows this result. When neat vapor is analyzed using the APCI source when lab air is of $50 \%$ to $60 \%$ relative humidity, the spectra match the "average" APPIS mass spectra (Figure 3b). An APCI mass spectrum of triethylamine using dry, compressed air (Figure 3c) yields triethylamine n-oxide as the most abundant ion with a significant percentage of protonated triethylamine. The formation of triethylamine n-oxide is likely a consequence of the redox conditions present during discharge, and others have observed similar addition of oxygen to triethylamine under oxidative conditions [21, 22]. Minor products observed $(m / z 72.1,74.1)$ are decomposition products of triethylamine n-oxide and are unique to that compound. These follow reactivity outlined by Ma and coworkers [23] of thermally induced decomposition of TEA-O during APCI. One additional minor product in the spectrum $(116.0 \mathrm{~m} / \mathrm{z})$ is identified via a reaction pathway analogous
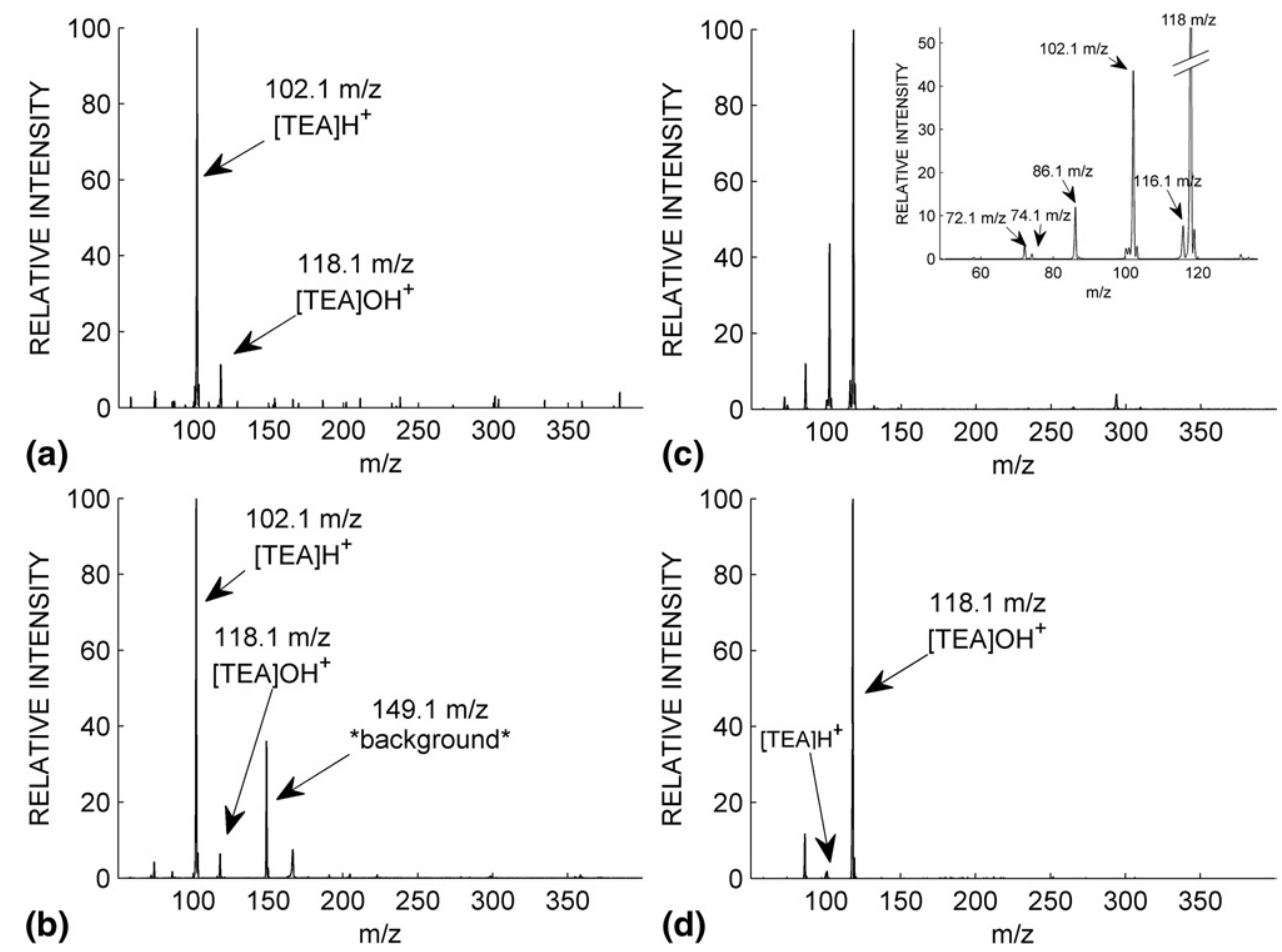

Figure 3. (a) APPIS mass spectrum of triethylamine with ambient air at approximately $40 \%$ to $50 \%$ relative humidity. This is a typical APPIS spectrum in that it is consistent with previously measured spectra, and is free from sample oxidation artifacts. (b) APCI mass spectrum of triethylamine, where neat vapor is allowed near the corona discharge in ambient air of moderate humidity. Note the close match to (a), indicating that in APCI the humidity of the air near the corona is important in determining the ionization products. The peak at $149.1 \mathrm{~m} / \mathrm{z}$ appears without sample vapor present as well, implicating it as an unknown background contaminant in lab air. (c) APCI mass spectrum of triethylamine using pneumatic air ejector for sample injection. Air is relatively dry around the corona due to use of compressed air as a carrier gas. (d) APPIS mass spectrum of triethylamine with ambient air at approx. $20 \%$ relative humidity, as determined by dew point and wet bulb thermometer measurements. Air is dry near the crystal. 
to that summarized by Atkinson [24], whereby triethylamine reacts with hydroxyl radical and through subsequent carbon and oxygen centered radical intermediates the species $\mathrm{CH}_{3} \mathrm{CON}\left(\mathrm{C}_{2} \mathrm{H}_{5}\right)_{2}$, or $\mathrm{N}, \mathrm{N}$-diethylacetamide is formed. Having a molecular weight of $115 \mathrm{Da}$, the protonated $\mathrm{N}, \mathrm{N}$-diethylacetamide is observed at $116.0 \mathrm{~m} / \mathrm{z}$. These oxidation products are formed along with the protonated species during ionization. Relative humidity of the air around the source region will determine the concentrations of reagent ions and thus the ratio of ionization products. We observe that with moderately humid conditions, the product from direct protonation is more likely to be observed than the oxidation products. The water clusters are dominant with high humidity, whereas the oxidative reagents play a prominent role with low humidity. On days when relative humidity in the lab is low (20\% to $30 \%$ relative humidity) the APPIS mass spectra in lab air nearly match the APCI mass spectra using dry compressed air (Figure $3 d$ ). This modulation of product distributions by ambient gas composition not only indicates that ionization by APPIS involves gas-phase processes, similar to APCI, but also that this source parameter, relative humidity of the gas surrounding the source, must be considered in interpreting source response.

We suggest an ionization mechanism involving observed electrical discharges near the face of the crystal resulting in reagent ions, which take part in chemical ionization reactions with analytes to result in the observed ionization products. Figure $1 \mathrm{~b}$ is a cartoon illustrating the proposed sequence of events leading to observation of an ion in the mass spectrometer. An excess of electrical charge is developed on the crystal face as a result of temperature cycling, and this charge is neutralized through electrical discharges either to the opposite crystal face, a region on the face of lesser charge due to uneven heating of the sample or crystal defects that lead to non-uniform charging of the surface, or a nearby grounded electrode. The transient signals from these discharges are measured using the inductive pickup fitted to the ionization source. Highly reactive and short-lived products resulting from the discharge react with molecules in ambient air to form the reagent precursor ions, which are directly observed in the ion trap mass spectrometer. Figure $4 \mathrm{a}$ and $\mathrm{b}$ show some of the reactant ions observed using APPIS in both anionic and cationic modes, with the mass spectrometer inlet capillary temperature reduced to $70{ }^{\circ} \mathrm{C}$. In particular, we directly observe ${ }^{-} \mathrm{OH}\left(\mathrm{H}_{2} \mathrm{O}\right)_{4}$ and ${ }^{+} \mathrm{H}\left(\mathrm{H}_{2} \mathrm{O}\right)_{5}$. These reactant ions are expected to undergo proton transfer reactions with neutral acidic and basic molecules, resulting in the observation of anionic and cationic species, respectively, in the mass spectrometer.

In addition to investigating the mechanism of ionization of APPIS, we have continued to examine the range of substances that are ionizable with the pyroelectric ion source, as well as the performance characteristics of the source. APPIS is a particularly simple and robust ion source, which lends itself to application
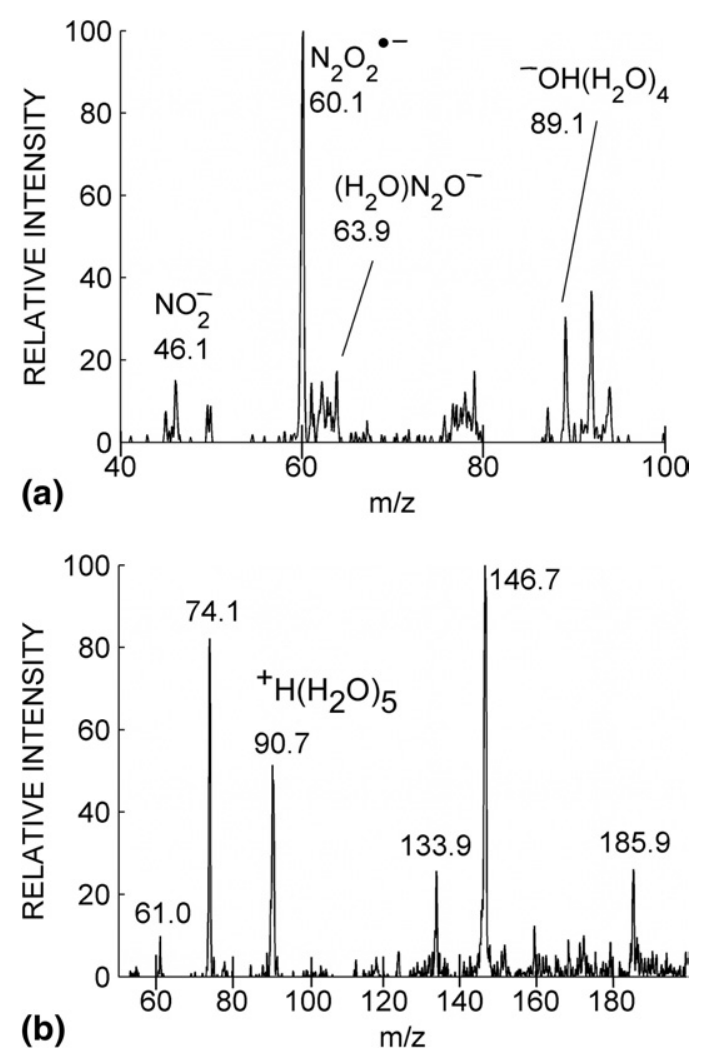

Figure 4. (a) APPIS mass spectrum of laboratory air, anionic mode. Reagent precursor ions, which can lead to gas-phase chemical ionization, are indicated. (b) APPIS mass spectrum of laboratory air, positive mode. The reagent precursor ion ${ }^{+} \mathrm{H}\left(\mathrm{H}_{2} \mathrm{O}\right)_{5}$ is noted at $90.7 \mathrm{~m} / \mathrm{z}$.

in harsh environments. One important application of an ion source with these characteristics is the detection of chemical warfare agents. We have examined simulants for chemical nerve agents in the $V$ and $G$ classes since they possess amine functionality, which has been shown to be advantageous for ionization using APPIS. Due to risks posed in working directly with these agents, compounds which simulate the reactivity of these agents are used instead. Hill and coworkers have used a number of these simulants in ion mobility experiments [18-20]. 2-(Butylamino)-ethanethiol (BAET), which simulates the nerve agent VX, and diethylphosophoramidate (DEPA), which simulates Tabun, were tested in the laboratory using APPIS. Table 1 summarizes the ability of APPIS to ionize these compounds, which were detected as protonated molecular ions using the ion trap mass spectrometer (Figure 5). Although the simulant for Tabun has no amine functionality, the protonated phosphodiester was nonetheless detected. In this experiment, the sample container was heated to produce significant vapor pressure of the simulants. The APPIS mass spectra for these compounds match the positive ion electrospray mass spectra. For BAET, a significant quantity of dimeric BAET was detected in both APPIS and electrospray mass spectra. This may result from high simulant concentrations and appropriate redox 
Table 1. Summary for the detection of CBW agent simulants by APPIS

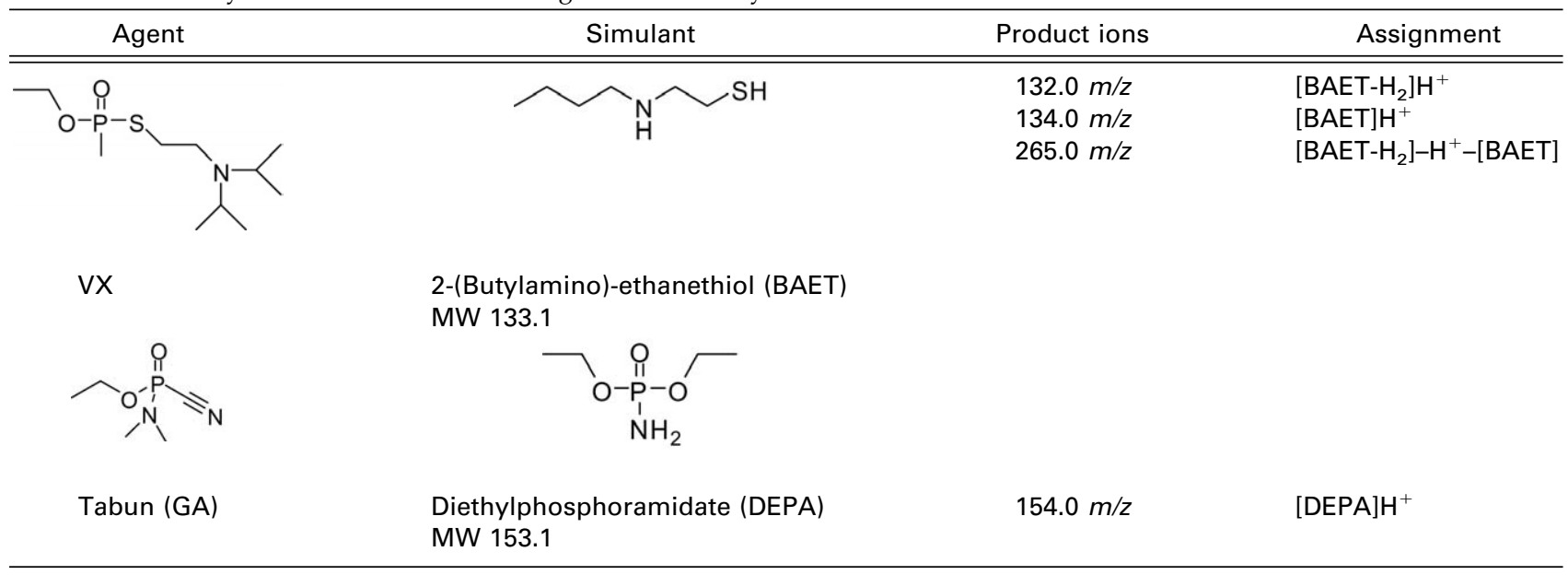

conditions at the time of ionization. Collision induced dissociation (CID) experiments verify the assignments given in Table 1.

Sample dilution experiments were performed to determine the performance and detection limits for APPIS. Pyridine was chosen as a test compound for performance evaluation. Samples of pyridine vapor were prepared in Tedlar sample bags and analyzed using APPIS. Figure 6 shows the detection of pyridine at a concentration of $4 \mathrm{ppm}$ in nitrogen. Water was added to the sample bag as the reagent molecule and is the proton source in this experiment. Detection of pyridine at a concentration of $4 \mathrm{ppm}$ with a signal-to-noise ratio of $\sim 10$ indicates that the ultimate sensitivity of APPIS for this compound, in the current source configuration, is likely in the high part per billion (ppb) range.

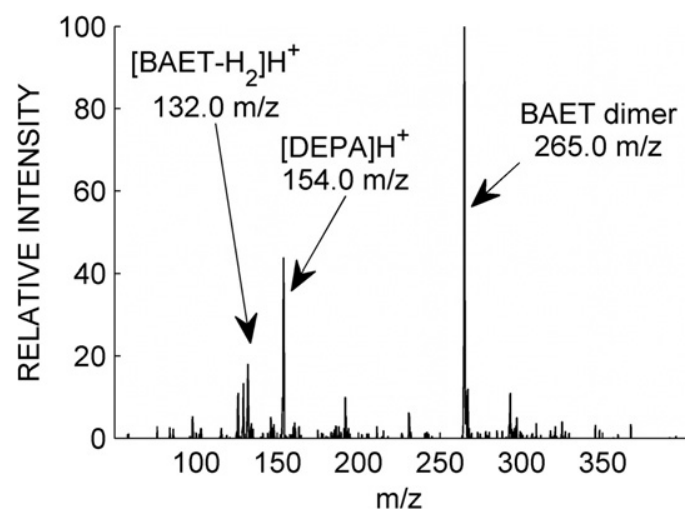

Figure 5. Mass spectrum showing the detection of chemical nerve agent simulants with APPIS. The infused sample was 2-(butylamino)ethanethiol (BAET, simulant for VX, a member of the $\mathrm{V}$ agent class) and diethyl phosphoramidate (DEPA, simulant for Tabun, member of the $G$ agent class). The protonated species are observed in the spectrum, with minimal fragmentation, allowing for the detection of signature masses of the stimulants, which were detected either as singly protonated species or dimeric complexes in APPIS mass spectra. Table 1 lists specific identities for each peak.

\section{Conclusion}

Studies of the ionization mechanism operative with APPIS reveal that ion formation involves a gas-phase process, occurring concurrently with electrical discharge activity, on the surface of the crystal as it is thermally cycled. For both positive and negative ions, reactions occurring between gases surrounding the ionization source and the analyte molecules are responsible for ion production. Ionization with APPIS is sensitive to environmental factors, specifically the ambient concentration of water vapor at the time of spectrum acquisition. Low relative humidity $\left(20 \%-40 \%, p\left(\mathrm{H}_{2} \mathrm{O}\right)=4\right.$ torr or $530 \mathrm{~Pa}$ ) yields ionization products that include target molecules oxidized by reactive oxygen species, whereas moderate humidity $\left(40 \%-70 \%, p\left(\mathrm{H}_{2} \mathrm{O}\right)=10\right.$ torr or $1.3 \times 10^{3} \mathrm{~Pa}$ ) yields primarily singly protonated cations or singly deprotonated anions. Mass spectra obtained with APPIS are similar to those recorded with atmospheric pressure chemical ionization using a corona discharge, further supporting the conjecture that electrical discharges are responsible for ion formation

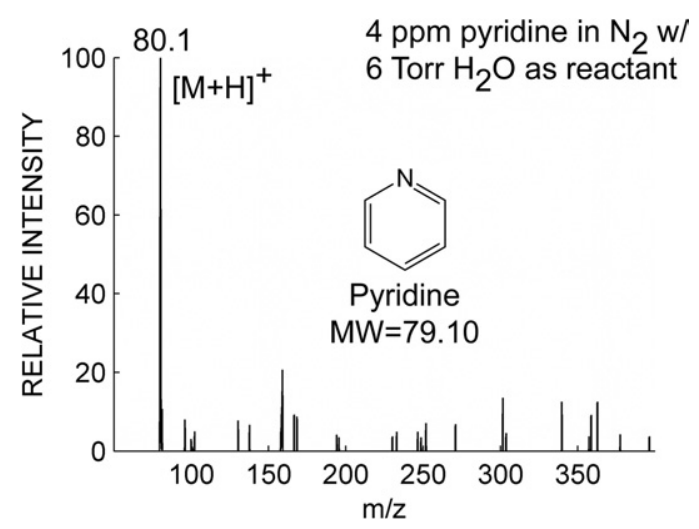

Figure 6. Mass spectrum of $4 \mathrm{ppm}$ pyridine in nitrogen, with 6 Torr $\mathrm{H}_{2} \mathrm{O}$ as reagent gas. Good signal to noise for the measurement indicates APPIS has an ultimate sensitivity for this chemical down to high part per billion range. 
with APPIS. To extend the applications of APPIS, chemical nerve agent simulants were analyzed. The species 2-(butylamino)-ethanethiol (simulant for VX, member of the $\mathrm{V}$ agent class) and diethyl phosphoramidate (simulant for Tabun, member of the $G$ agent class) were detected either as singly protonated species or dimeric complexes in APPIS mass spectra, suggesting possible future use of an optimized source for the detection of volatile chemical warfare agents. Pyridine was readily detected down to concentrations of $4 \mathrm{ppm}$, suggesting ultimate sensitivity of APPIS for a fully optimized system in the high part-per-billion range.

\section{Acknowledgments}

The authors acknowledge funding from the National Science Foundation under grant CHE-0416381 as well funding from the Beckman Institute at Caltech. ELN acknowledges a Graduate Research Fellowship in Analytical Chemistry from Pfizer Analytical Research and Development (Groton, CT), as well as many useful discussions with Luther Beegle (Jet Propulsion Laboratory, Pasadena, CA), Jim Weiss (Ionfinity LLC, Walnut, CA), and Professor G. A. Eiceman (New Mexico State University, Las Cruces, NM).

\section{References}

1. Neidholdt, E. L.; Beauchamp, J. L. Ambient pressure pyroelectric ion source for mass spectrometry. Anal. Chem. 2007, 79, 3945-3948.

2. McLuckey, S. A.; Glish, G. L.; Asano, K. G.; Grant, B. C. Atmospheric sampling glow discharge ionization source for the determination of trace organic compounds in ambient air. Anal. Chem. 1988, 60, $2220-$ 2227.

3. Harrison, W. W.; Hess, K. R.; Marcus, R. K.; King, F. L. Glow discharge mass spectrometry. Anal. Chem. 1986, 58, 341A-356A.

4. Mason, R.; Milton, D. Glow discharge mass spectrometry of some organic compounds. Int. J. Mass Spectrom. Ion Processes 1989, 91, 209225 .

5. Carroll, D. I.; Dzidic, I.; Stillwell, R. N.; Haegele, K. D.; Horning, E. C. Atmospheric pressure ionization mass spectrometry: Corona discharge ion source for use in liquid chromatograph-mass spectrometer-computer analytical system. Anal. Chem. 1975, 47, 2369-2373.

6. Na, N.; Zhao, M.; Zhang, S.; Yang, C.; Zhang, X. Development of a dielectric barrier discharge ion source for ambient mass spectrometry. J. Am. Soc. Mass Spectrom. 2007, 18, 1859-1862.
7. Bayssie, M.; Brownridge, J. D.; Kukhtarev, N.; Wang, J. C. Generation of focused electron beam and $\mathrm{X}$-rays by the doped $\mathrm{LiNbO} 3$ crystals. $\mathrm{Nucl}$. Instrum. Methods Phys. Res. B 2005, 241, 913-916.

8. Geuther, J. A.; Danon, Y. Electron and positive ion acceleration with pyroelectric crystals. J. Appl. Phys. 2005, 97, 074109.

9. Brownridge, J. D.; Shafroth, S. M. Using static charge on pyroelectric crystals to produce self-focusing electron and ion beams and transport through tubes. J. Electrostat. 2005, 63, 249-259.

10. Kukhtarev, N.; Kukhtareva, J. D. T.; Bayssie, M.; Wang, J.; Brownridge, J. D. Generation of focused electron beam by pyroelectric and photogalvanic crystals. J. Appl. Phys. 2004, 96, 6794-6798.

11. Brownridge, J. D.; Shafroth, S. M. Pressure dependence of energetic $(\leq 160 \mathrm{keV})$ focused electron beams arising from heated or cooled (LiNbO3) pyroelectric crystals. Appl. Phys. Lett. 2003, 83, 1477-1479.

12. Brownridge, J. D.; Shafroth, S. M. Self-focused electron beams produced by pyroelectric crystals on heating or cooling in dilute gases. Appl. Phys. Lett. 2001, 79, 3364-3366.

13. Brownridge, J. D.; Shafroth, S. M.; Trott, D. W.; Stoner, B. R.; Hooke, W. M. Observation of multiple nearly monoenergetic electron production by heated pyroelectric crystals in ambient gas. Appl. Phys. Lett. 2001, $78,1158-1159$.

14. Rosenblum, B.; Braulich, P.; Carrico, J. P. Thermally stimulated field emission from pyroelectric lithium niobate. Appl. Phys. Lett. 1974, 25, 17-19.

15. Geuther, J.; Danon, Y. High-energy X-ray production with pyroelectric crystals. J. Appl. Phys. 2005, 97, 104916.

16. Naranjo, B.; Gimzewski, J. K.; Putterman, S. Observation of nuclear fusion driven by a pyroelectric crystal. Nature 2005, 434, 1115-1117.

17. Geuther, J.; Danon, Y.; Saglime, F. Nuclear reactions induced by a pyroelectric accelerator. Phys. Rev. Lett. 2006, 96, 054803.

18. Steiner, W. E.; Clowers, B. H.; Haigh, P. E.; Hill, H. H. Secondary ionization of chemical warfare agent simulants: Atmospheric pressure ion mobility time-of-flight mass spectrometry. Anal. Chem. 2003, 75, 6068-6076.

19. Steiner, W. E.; English, W. A.; Hill, H. H. Separation efficiency of a chemical warfare agent simulant in an atmospheric pressure ion mobility time-of-flight mass spectrometer (IM(TOF)MS). Anal. Chim. Acta 2005, 532, 37-45.

20. Steiner, W. E.; Klopsch, S. J.; English, W. A.; Clowers, B. H.; Hill, H. H. Detection of a chemical warfare agent simulant in various aerosol matrixes by ion mobility time-of-flight mass spectrometry. Anal. Chem. 2005, 77, 4792-4799.

21. Riley, D. P. Ruthenium chloride catalyzed oxidation of tertiary amines to amine oxides with molecular oxygen. J. Chem. Soc., Chem. Commun. 1983, 24, 1530-1532.

22. Riley, D. P.; Correa, P. E. An unprecedented selective autoxidation of tertiary amines to amine oxides. J. Org. Chem. 1985, 50, 1564-1566.

23. Ma, S.; Chowdhury, S. K.; Alton, K. B. Thermally induced n-to-o rearrangement of tert-N-oxides in atmospheric pressure chemical ionization and atmospheric pressure photoionization mass spectrometry: Differentiation of N-oxidation from hydroxylation and potential determination of N-oxidation site. Anal. Chem. 2005, 77, 3676-3682.

24. Atkinson, R. Kinetics and mechanisms of the gas-phase reactions of the hydroxyl radical with organic compounds under atmospheric conditions. Chem. Rev. 1985, 85, 69-201. 